\title{
Temperatura na germinação de sementes de genótipos de pitaya
}

\section{Temperature in the seeds germination of pitaya genotypes}

\author{
Alessandro Borini Lone ${ }^{1 *}$; Ronan Carlos Colombo ${ }^{2}$; Vanessa Favetta ${ }^{2}$; \\ Lúcia Sadayo Assari Takahashi ${ }^{3}$; Ricardo Tadeu de Faria ${ }^{4}$
}

\section{Resumo}

As temperaturas ótimas para a germinação de sementes de cactos variam com a espécie. Com esse trabalho, objetivou-se avaliar a germinação de sementes de genótipos de pitaya sob diferentes temperaturas. Os genótipos utilizados foram: Hylocereus undatus (PB), H. polyrhizus (PV), Selenicereus megalanthus (PA), H. undatus x H. costaricensis (PH1) e H. costaricensis x H. undatus (PH2). Para cada genótipo utilizou-se quatro repetições de 50 sementes, em delineamento experimental inteiramente casualizado. A semeadura foi realizada sobre papel mata-borrão, em caixas tipo Gerbox ${ }^{\circledR}$, mantidas sob as temperaturas de $15,20,25,30$ e $35{ }^{\circ} \mathrm{C}$ constantes e $15-25,20-30$ e $25-35{ }^{\circ} \mathrm{C}$ alternadas, com fotoperíodo de 12 horas. $\mathrm{O}$ teste de germinação durou 30 dias, nos quais foram avaliados a porcentagem, o tempo médio e o índice de velocidade de germinação. Para a germinação de sementes de PB, o resultado obtido na temperatura de $25^{\circ} \mathrm{C}$ não diferiu dos obtidos a 30 e $20-30^{\circ} \mathrm{C}$, no entanto foi superior às demais temperaturas. Em PV, o resultado a $25^{\circ} \mathrm{C}$ não diferiu dos obtidos à 20 e $30^{\circ} \mathrm{C}$, sendo superior aos resultados das demais temperaturas. Para PA, o melhor resultado foi obtido a $25^{\circ} \mathrm{C}$. Em PH1, as temperaturas de 25,30 e $20-30^{\circ} \mathrm{C}$ apresentaram resultados superiores às demais. Para $\mathrm{PH} 2$, o resultado obtido em $15-25^{\circ} \mathrm{C}$ não diferiu do obtido à $25^{\circ} \mathrm{C}$, no entanto foi superior aos das demais temperaturas. As temperaturas constantes de 25 e $30^{\circ} \mathrm{C}$ e a alternada de $20-30{ }^{\circ} \mathrm{C}$ são adequadas para a germinação de sementes de $H$. undatus e para o híbrido $H$. undatus x $H$. costaricensis. Para $H$. polyrhizus, as temperaturas constantes de 20,25 e $30^{\circ} \mathrm{C}$ são adequadas para a germinação das sementes. A temperatura constante de $25^{\circ} \mathrm{C}$ é a mais adequada para a germinação de sementes de S. megalanthus. Para o híbrido H. costaricensis $\mathrm{x} H$. undatus, a temperatura constante de $25^{\circ} \mathrm{C}$ e a alternada de $15-25^{\circ} \mathrm{C}$ são adequadas para a germinação das sementes.

Palavras-chave: Cactaceae, fruta-dragão, Hylocereus, Selenicereus, teste de germinação

\footnotetext{
Abstract

The optimum temperature for germination of cacti vary with the species. With this work, we aimed to evaluate the seeds germination of pitaya genotypes under different temperatures. The used genotypes were: Hylocereus undatus (PB), H. polyrhizus (PV), Selenicereus megalanthus (PA), H. undatus x H. costaricensis (PH1) and H. costaricensis $\mathrm{x} H$. undatus (PH2). For each genotype we used four replicates of 50 seeds, in a completely randomized design. The sowing was carried out on blotter paper in boxes type Gerbox ${ }^{\circledR}$, maintained at temperatures of $15,20,25,30$ and $35{ }^{\circ} \mathrm{C}$ constant and $15-25,20-30$ and $25-35{ }^{\circ} \mathrm{C}$ alternating with photoperiod 12 hours. The test lasted 30 days which were appraised the

${ }^{1}$ Biólogo, Dr. em Agronomia, Universidade Estadual de Londrina, UEL, Londrina, PR. E-mail: alone_bio@yahoo.com.br

${ }^{2}$ Eng $^{\text {os }}$ Agr $^{\text {os }}$, Discentes de Mestrado em Agronomia, UEL, Londrina, PR. Bolsistas da CAPES. E-mail: ronancolombo@yahoo. com.br; vanfavetta@hotmail.com

${ }^{3}$ Eng $^{\mathrm{a}}$ Agr $^{\mathrm{a}}$, Prof ${ }^{\mathrm{a}}$ Dr $^{\mathrm{a}}$ do Dept ${ }^{\mathrm{o}}$ de Agronomia, UEL, Londrina, PR. Bolsista Produtividade da Fundação Araucária. E-mail: sadayo@uel.br

${ }^{4}$ Eng $^{\mathrm{o}}$ Agr $^{\mathrm{o}}$, Prof. Dr. do Dept ${ }^{\mathrm{o}}$ de Agronomia, UEL, Londrina, PR. Bolsista Produtividade do CNPq. E-mail: faria@uel.br

* Autor para correspondência
} 
germination percentage, the germination speed index and the average time of germination. For seeds germination of $\mathrm{PB}$, the result obtained in the temperature of $25^{\circ} \mathrm{C}$ didn't differ of the obtained to 30 and 20-30 ${ }^{\circ} \mathrm{C}$, however it was superior to the others temperatures. In PV, the result at $25{ }^{\circ} \mathrm{C}$ didn't differ of the obtained to 20 and $30^{\circ} \mathrm{C}$, being superior to the results of the others temperatures. For PA, the best result was obtained to $25^{\circ} \mathrm{C}$. In $\mathrm{PH} 1$, the temperatures of 25,30 and $20-30{ }^{\circ} \mathrm{C}$ presented superiors results to the others. For PH2, the result obtained in $15-25^{\circ} \mathrm{C}$ didn't differ of the obtained at $25^{\circ} \mathrm{C}$, however it was superior to the others temperatures. The constants temperatures of 25 and $30^{\circ} \mathrm{C}$ and alternating 20 $30{ }^{\circ} \mathrm{C}$ are suitable for germination of $H$. undatus and for the hybrid $H$. undatus $\mathrm{x} H$. costaricensis. For H. polyrhizus, constant temperatures of 20,25 to $30^{\circ} \mathrm{C}$ are suitable for seed germination. The constant temperature of $25{ }^{\circ} \mathrm{C}$ is the most suitable for the germination of $S$. megalanthus. For the hybrid $H$. costaricensis $\mathrm{x} \mathrm{H}$. undatus, constant temperature of $25^{\circ} \mathrm{C}$ and alternating $15-25^{\circ} \mathrm{C}$ are suitable for seed germination.

Key words: Cactaceae, dragon fruit, Hylocereus, Selenicereus, germination test

\section{Introdução}

Pitaya é o nome dado aos frutos de diversas cactáceas de hábito trepador, os quais apresentam pequenas sementes digeríveis e casca que pode ter ou não espinhos, porém se desprendem espontaneamente durante a maturação (NERD; TEL-ZUR; MIZRAHI, 2002). Algumas espécies têm demonstrado serem aptas para a comercialização, dentre as quais se destacam a Hylocereus undatus (fruto oblongo, com casca vermelha e polpa branca), a Hylocereus polyrhizus (fruto oblongo, com casca vermelha e polpa vermelha), Hylocereus costaricenses (fruto globoso, com casca vermelha e polpa vermelha) e Selenicereus megalanthus (fruto oblongo, com casca amarela e polpa branca) (MIZRAHI; NERD; NOBEL, 1997; NERD; TELZUR; MIZRAHI, 2002).

As diversas espécies de pitaya são originárias do continente americano e se encontram distribuídas nos países da Costa Rica, Equador, Peru, Venezuela, Panamá, Uruguai, Brasil, Colômbia e México, sendo os dois últimos os principais produtores a nível mundial (CANTO, 1993; ANDERSON, 2001; VAILLANT et al., 2005).

A reprodução da pitaya pode ser de modo vegetativo ou por meio de sementes, a qual é conveniente, principalmente em programas de melhoramento, porque se obtém materiais com diferentes informações genéticas, apresentando características diversas que podem ser aproveitadas.
As plantas originadas por propagação sexuada apresentam, portanto, grande variabilidade, o que torna possível a seleção de genótipos com características desejáveis, tais como produtividade, aparência externa, coloração de polpa e melhor adaptação às diferentes condições climáticas (ANDRADE et al., 2008).

Estudos sobre a influência da temperatura na germinação são essenciais para entender os aspectos ecofisiológicos e bioquímicos desse processo; influenciados pelas condições ambientais às quais as sementes são expostas na germinação (LABOURIAU, 1983; MALUF; MARTINS, 1991; BEWLEY; BLACK, 1994). As temperaturas ótimas para a germinação de sementes de cactos variam com a espécie, sendo de $20^{\circ} \mathrm{C}$ para Schlumbergera truncata (flor-de-maio) (LONE et al., 2010), 25 ${ }^{\circ} \mathrm{C}$ para Melocactus bahiensis (coroa-de-frade) (LONE et al., 2007), e $30^{\circ} \mathrm{C}$ para Cereus jamacaru (Mandacaru) (GUEDES et al., 2009).

Sementes de determinadas espécies germinam melhor quando submetidas à alternância de temperatura, a qual corresponde às flutuações naturais encontradas no ambiente. No entanto, existem espécies em que a germinação de suas sementes é favorecida quando submetidas à temperatura constante (ALVES; GODOY; CORREA, 2011). Desse modo torna-se importante a inclusão de temperaturas alternadas e constantes no teste de germinação para a constatação das melhores 
condições para a germinação das sementes.

O objetivo do trabalho foi avaliar a germinação de sementes de genótipos de pitaya sob diferentes temperaturas.

\section{Material e Métodos}

O experimento foi realizado no laboratório de Fitotecnia da Universidade Estadual de Londrina (UEL) - PR, utilizando sementes de genótipos de pitaya extraídas de frutos obtidos em plantas matrizes existentes na Fazenda Escola da UEL. Os genótipos utilizados foram compostos de três espécies e dois híbridos: Hylocereus undatus (Haworth) Britton \& Rose (PB), Hylocereus polyrhizus (F.A.C. Weber) Britton \& Rose (PV), Selenicereus megalanthus (K.Schum. ex Vaupel) Moran) (PA), (Hylocereus undatus x Hylocereus costaricensis (PH1) e Hylocereus costaricensis $\mathrm{x}$ Hylocereus undatus (PH2).

Para a extração das sementes empregou-se o processo de fermentação da polpa por 48 horas, em solução de água + açúcar 2,5\%. Após esse período, a solução foi peneirada em água corrente de modo a eliminar os resíduos da polpa e reter somente as sementes as quais foram colocadas para secar à sombra, sobre papel de filtro, durante 48 horas em temperatura ambiente. As sementes foram acondicionadas em embalagens de polietileno e armazenadas durante 20 dias em câmara fria (temperatura de $6-9{ }^{\circ} \mathrm{C}$ e $75 \%$ de umidade relativa do ar).

Folhas de papel mata-borrão foram umedecidas com água destilada, na quantidade de 2,5 vezes a massa do papel não hidratado (BRASIL, 2009) acondicionadas em caixas plásticas transparentes com tampa (Gerbox $\left.{ }^{\circledR}\right)$. Em cada caixa foram semeadas 50 sementes $\mathrm{e}$ as mesmas foram acondicionadas sob as temperaturas constantes de $15,20,25,30$ e $35^{\circ} \mathrm{C}$ e as alternadas de $15-25,20$ 30 e $25-35^{\circ} \mathrm{C}$ em fotoperíodo de $12 \mathrm{~h}$, e intensidade luminosa de $16,6 \mathrm{~mol} \mathrm{~m} \mathrm{~m}^{-2} \mathrm{~s}^{-1} \pm 0,15$, condições obtidas com a utilização de germinadores. $\mathrm{O}$ delineamento experimental foi o inteiramente casualizado com quatro repetições por tratamento para cada genótipo, sendo cada genótipo analisado separadamente.

As avaliações do teste de germinação ocorreram por meio de contagens diárias durante 30 dias. Considerou-se germinadas, as sementes que emitiram o hipocótilo. As variáveis avaliadas foram a porcentagem de germinação, o índice de velocidade de germinação (IVG), calculado de acordo com a fórmula de Maguire (1962), descrita por Nakagawa (1994): IVG $=(\mathrm{G} 1 / \mathrm{N} 1)+(\mathrm{G} 2 / \mathrm{N} 2)$ $+\ldots+(\mathrm{Gn} / \mathrm{Nn})$, em que:

$\mathrm{G} 1=$ número de sementes germinadas na primeira contagem;

$\mathrm{N} 1$ = número de dias decorridos até a primeira contagem;

$\mathrm{G} 2$ = número de sementes germinadas na segunda contagem;

$\mathrm{N} 2$ = número de dias decorridos até a segunda contagem;

$\mathrm{n}=$ última contagem,

e o tempo médio de germinação (TMG - dias), calculado segundo Lima et al. (2006): TMG = $(\Sigma$ niti)/ $/$ ni, em que:

ni = número de sementes germinadas por dia;

ti = tempo de incubação;

$\mathrm{i}=1 \rightarrow 30$ dias. Unidade: dias.

Os valores de porcentagem de germinação foram transformados em arco seno $\sqrt{ }(\mathrm{x} / 100)^{0,5}$. Os dados foram submetidos a análise de variância, seguida do teste de Tukey a 5\% de significância, para comparação das médias; utilizando-se o software estatístico SASM Agri (CANTERI et al., 2001).

\section{Resultados e Discussão}

A porcentagem de germinação de $\mathrm{PB}$, sob as 
temperaturas constantes de 15 a $30^{\circ} \mathrm{C}$ e as alternadas 15-25 e $20-30{ }^{\circ} \mathrm{C}$, apresentou valores superiores em detrimento à 35 e $25-35^{\circ} \mathrm{C}$. Em PV, apenas sob a temperatura de $35^{\circ} \mathrm{C}$, a porcentagem de germinação foi inferior às demais. Em PA também se observou valores inferiores de germinação sob 35 e 25-35
${ }^{\circ} \mathrm{C}$. Nesse caso, a temperatura de $35{ }^{\circ} \mathrm{C}$, pode estar próxima à temperatura limite para a germinação de sementes dessas espécies (Tabela 1). Segundo Marcos Filho (1986) as temperaturas máximas para a germinação de sementes da maioria das plantas cultivadas estão entre 35 e $40{ }^{\circ} \mathrm{C}$.

Tabela 1. Germinação (\%) de sementes de pitaya das espécies H. undatus (PB), H. polirhyzus (PV) e S. megalanthus (PA), e dos híbridos H. undatus $x H$. costaricensis (PH1) e H. costaricensis $x H$. undatus (PH2) em diferentes temperaturas $\left({ }^{\circ} \mathrm{C}\right)$.

\begin{tabular}{cccccc}
\hline Temp. $\left({ }^{\circ} \mathrm{C}\right)$ & PB & PV & PA & PH1 & PH2 \\
\hline 15 & $95 \mathrm{a}$ & $86 \mathrm{a}$ & $75 \mathrm{ab}$ & $73^{\mathrm{ns}}$ & 97 ns \\
20 & $97 \mathrm{a}$ & $91 \mathrm{a}$ & $69 \mathrm{ab}$ & 81 & 97 \\
25 & $98 \mathrm{a}$ & $91 \mathrm{a}$ & $88 \mathrm{a}$ & 85 & 99 \\
30 & $92 \mathrm{a}$ & $86 \mathrm{a}$ & $70 \mathrm{ab}$ & 78 & 96 \\
35 & $50 \mathrm{~b}$ & $40 \mathrm{~b}$ & $30 \mathrm{c}$ & 80 & 94 \\
$15-25$ & $97 \mathrm{a}$ & $85 \mathrm{a}$ & $63 \mathrm{~b}$ & 76 & 99 \\
$20-30$ & $93 \mathrm{a}$ & $80 \mathrm{a}$ & $70 \mathrm{ab}$ & 81 & 97 \\
$25-35$ & $30 \mathrm{~b}$ & $82 \mathrm{a}$ & $22 \mathrm{c}$ & 72 & 99 \\
\hline $\mathrm{CV}(\%)$ & 9,88 & 6,53 & 14,05 & 6,63 & 7,13 \\
\hline
\end{tabular}

Médias seguidas da mesma letra, na coluna, não diferem entre si pelo teste de Tukey a 5\% de probabilidade.

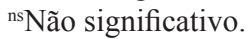

Fonte: Elaboração dos autores.

No entanto, nos híbridos avaliados (PH1 e PH2), não se observaram diferenças para germinação entre as temperaturas avaliadas, indicando que a temperatura limite para a germinação das sementes destes, provavelmente, são superiores a $35{ }^{\circ} \mathrm{C}$. Simão, Socolowski e Takaki (2007) verificaram porcentagens de germinação superiores a $70 \%$ para sementes de Hylocereus setaceus (Cactaceae) submetidas a temperaturas entre 15 e $40{ }^{\circ} \mathrm{C}$, atingindo $100 \%$ sob temperatura de $25^{\circ} \mathrm{C}$. Enquanto, Guedes et al. (2009), verificaram em trabalho com germinação de sementes de Cereus jamacaru (Cactaceae) que a temperatura de $30{ }^{\circ} \mathrm{C}$ foi a que apresentou os melhores resultados, em relação à 25 ${ }^{\circ} \mathrm{C}$ e à $20-30{ }^{\circ} \mathrm{C}$.

Para o IVG, em PB a temperatura de $25^{\circ} \mathrm{C}$ resultou em média significativamente superior às temperaturas de $30^{\circ} \mathrm{C}$ e $20-30{ }^{\circ} \mathrm{C}$. Em PV a temperatura de $25^{\circ} \mathrm{C}$ também foi a que apresentou a maior média, não diferindo dos valores das temperaturas de 20 e $30^{\circ} \mathrm{C}$. Em PA, a temperatura de $25{ }^{\circ} \mathrm{C}$ resultou em valor superior para IVG em relação às demais temperaturas. Para as espécies avaliadas observou-se que as temperaturas de 35 e $25-35{ }^{\circ} \mathrm{C}$ proporcionaram menores valores para IVG. Para PH1 as temperaturas de 25, 30 e 20$30{ }^{\circ} \mathrm{C}$ resultaram em maiores valores de IVG em relação às demais temperaturas enquanto em $\mathrm{PH} 2$, a temperatura de $15-25^{\circ} \mathrm{C}$ proporcionou maior valor médio de IVG, não diferindo da média obtida na temperatura de $25^{\circ} \mathrm{C}$. Observou-se que para os dois híbridos avaliados (PH1 e $\mathrm{PH} 2$ ) a temperatura de $15{ }^{\circ} \mathrm{C}$ foi a que proporcionou os menores valores médios de IVG (Tabela 2).

A temperatura influi na velocidade de absorção de água e modifica a velocidade das reações químicas que irão mobilizar ou degradar as reservas armazenadas e a síntese de várias substâncias para 
o crescimento das plântulas (BEWLEY; BLACK, 1994). Desse modo, baixas temperaturas podem reduzir a velocidade dessas reações, reduzindo a velocidade de germinação de sementes, como verificado a $15{ }^{\circ} \mathrm{C}$ para todos os genótipos de pitaya, e temperaturas altas podem interferir na síntese protéica também ocasionando redução na velocidade de germinação (HENDRICKS; TAYLORSON, 1976; RILEY, 1981), como verificado a 35 e $25-35^{\circ} \mathrm{C}$.

Tabela 2. Índice de velocidade de germinação (IVG) de sementes de pitaya das espécies H. undatus (PB), H. polirhyzus (PV) e S. megalanthus (PA), e dos híbridos H. undatus $x$ H. costaricensis (PH1) e H. costaricensis $x$ H. undatus (PH2) em diferentes temperaturas $\left({ }^{\circ} \mathrm{C}\right)$.

\begin{tabular}{ccrrrr}
\hline Temp. $\left({ }^{\circ} \mathrm{C}\right)$ & PB & PV & PA & PH1 & PH2 \\
\hline 15 & $3,63 \mathrm{c}$ & $6,61 \mathrm{~d}$ & $3,84 \mathrm{~cd}$ & $4,19 \mathrm{~d}$ & $5,71 \mathrm{e}$ \\
20 & $5,78 \mathrm{~b}$ & $10,92 \mathrm{ab}$ & $5,37 \mathrm{c}$ & $7,02 \mathrm{~b}$ & $9,44 \mathrm{~d}$ \\
25 & $7,29 \mathrm{a}$ & $12,37 \mathrm{a}$ & $9,16 \mathrm{a}$ & $10,28 \mathrm{a}$ & $15,15 \mathrm{ab}$ \\
30 & $6,69 \mathrm{ab}$ & $11,20 \mathrm{ab}$ & $5,37 \mathrm{c}$ & $10,37 \mathrm{a}$ & $14,86 \mathrm{bc}$ \\
35 & $2,49 \mathrm{~d}$ & $3,65 \mathrm{e}$ & $1,48 \mathrm{e}$ & $7,32 \mathrm{~b}$ & $9,93 \mathrm{~d}$ \\
$15-25$ & $3,99 \mathrm{c}$ & $8,01 \mathrm{~cd}$ & $3,40 \mathrm{~d}$ & $4,70 \mathrm{~cd}$ & $16,48 \mathrm{a}$ \\
$20-30$ & $6,39 \mathrm{ab}$ & $9,99 \mathrm{bc}$ & $7,12 \mathrm{~b}$ & $9,31 \mathrm{a}$ & $13,98 \mathrm{bc}$ \\
$25-35$ & $1,35 \mathrm{e}$ & $9,17 \mathrm{bc}$ & $0,92 \mathrm{e}$ & $5,89 \mathrm{bc}$ & $13,49 \mathrm{c}$ \\
\hline $\mathrm{CV}(\%)$ & 9,73 & 10,60 & 15,41 & 9,31 & 5,35 \\
\hline
\end{tabular}

Médias seguidas da mesma letra, na coluna, não diferem entre si pelo teste de Tukey a 5\% de probabilidade.

Fonte: Elaboração dos autores.

Em relação ao tempo médio de germinação (TMG), em PB a menor média (7,1 dias), caracterizando uma germinação mais rápida, foi obtida na temperatura de $25^{\circ} \mathrm{C}$, não diferindo das temperaturas de 30 e $20-30{ }^{\circ} \mathrm{C}$. Para essa espécie os TMGs variaram de 7,1 dias em $25^{\circ} \mathrm{C}$ a 13,5 dias em $15^{\circ} \mathrm{C}$. Em PV as médias variaram entre 4,4 dias em $25^{\circ} \mathrm{C}$ a 7,0 dias em $15^{\circ} \mathrm{C}$, com os menores tempos médios de germinação observados a 20 e $25^{\circ} \mathrm{C}(4,5$ e 4,4 dias), não diferindo da temperatura de $30{ }^{\circ} \mathrm{C}$ (4,9 dias). Em PA o menor TMG foi observado em $25^{\circ} \mathrm{C}$ (5,3 dias), não diferindo das médias das temperaturas de 20 e $20-30{ }^{\circ} \mathrm{C}$. Em PH1 os melhores resultados foram obtidos nas temperaturas de 25 , 30, e $20-30{ }^{\circ} \mathrm{C}$, com médias de 4,5, 4,2 e 5,0 dias respectivamente. Em $\mathrm{PH} 2$ o menor valor de TMG foi observado na temperatura de $15-25^{\circ} \mathrm{C}$ (2,2 dias), não diferindo das médias das temperaturas de 25 , 30 e 20-30 ${ }^{\circ} \mathrm{C}$. Para os híbridos, a temperatura de 15 ${ }^{\circ} \mathrm{C}$, acrescida da $15-25{ }^{\circ} \mathrm{C}$ para $\mathrm{PH} 1$, foram as que apresentaram as germinações mais tardias, ficando entre 8,6 e 9,4 dias em 15 e $15-25^{\circ} \mathrm{C}$ para $\mathrm{PH} 1$, e de 7,8 dias em $15^{\circ} \mathrm{C}$ para $\mathrm{PH} 2$ (Tabela 3).

Em seu trabalho com germinação de Melocactus bahiensis (Cactaceae), Lone et al. (2007), obtiveram valores de TMG que variaram de 16 dias a $20{ }^{\circ} \mathrm{C}$, 7,5 dias a $25^{\circ} \mathrm{C}$, até 7,1 a $30^{\circ} \mathrm{C}$, valores próximos aos encontrados no presente trabalho para PB em 25 e $30^{\circ} \mathrm{C}$ (7,1 e 7,6 dias, respectivamente) e para PA a $30^{\circ} \mathrm{C}(7,6$ dias $)$.

Para o teste de germinação de sementes dos genótipos de pitaya, verificou-se que os melhores resultados foram obtidos com temperaturas em torno de $25{ }^{\circ} \mathrm{C}$, apresentando certa variação entre as espécies e os híbridos. Segundo Carvalho e Nakagawa (2000), a germinação só ocorre dentro de determinados limites de temperatura, nos quais existe uma temperatura ótima, ou faixa de temperatura na qual o processo ocorre com a máxima eficiência, obtendo-se o máximo de germinação no menor período possível. 
Tabela 3. Tempo médio de germinação (dias) de sementes de pitaya das espécies H. undatus (PB), H. polirhyzus (PV) e S. megalanthus (PA), e dos híbridos H. undatus $x$ H. costaricensis (PH1) e H. costaricensis $x$ H. undatus (PH2) em diferentes temperaturas $\left({ }^{\circ} \mathrm{C}\right)$.

\begin{tabular}{crrrrc}
\hline Temp. $\left({ }^{\circ} \mathrm{C}\right)$ & PB & PV & PA & PH1 & PH2 \\
\hline 15 & $13,47 \mathrm{~d}$ & $7,03 \mathrm{e}$ & $11,49 \mathrm{~cd}$ & $9,44 \mathrm{c}$ & $7,82 \mathrm{~d}$ \\
20 & $8,69 \mathrm{~b}$ & $4,54 \mathrm{a}$ & $7,25 \mathrm{ab}$ & $6,23 \mathrm{~b}$ & $4,30 \mathrm{c}$ \\
25 & $7,11 \mathrm{a}$ & $4,39 \mathrm{a}$ & $5,28 \mathrm{a}$ & $4,48 \mathrm{a}$ & $2,31 \mathrm{ab}$ \\
30 & $7,57 \mathrm{ab}$ & $4,86 \mathrm{ab}$ & $7,56 \mathrm{~b}$ & $4,21 \mathrm{a}$ & $2,30 \mathrm{ab}$ \\
35 & $11,10 \mathrm{c}$ & $6,39 \mathrm{de}$ & $10,51 \mathrm{~cd}$ & $6,51 \mathrm{~b}$ & $4,07 \mathrm{c}$ \\
$15-25$ & $12,39 \mathrm{~d}$ & $6,13 \mathrm{~cd}$ & $9,74 \mathrm{c}$ & $8,61 \mathrm{c}$ & $2,16 \mathrm{a}$ \\
$20-30$ & $7,96 \mathrm{ab}$ & $4,93 \mathrm{ab}$ & $6,13 \mathrm{ab}$ & $5,04 \mathrm{a}$ & $2,56 \mathrm{ab}$ \\
$25-35$ & $13,16 \mathrm{~d}$ & $5,56 \mathrm{bc}$ & $12,47 \mathrm{~d}$ & $7,17 \mathrm{~b}$ & $2,81 \mathrm{~b}$ \\
\hline $\mathrm{CV}(\%)$ & 5,38 & 6,03 & 9,87 & 6,28 & 7,37 \\
\hline
\end{tabular}

Médias seguidas da mesma letra, na coluna, não diferem entre si pelo teste de Tukey a 5\% de probabilidade.

Fonte: Elaboração dos autores.

Verificou-se também que a temperatura de 35 ${ }^{\circ} \mathrm{C}$ não foi favorável para germinação de sementes dos genótipos de pitaya avaliados. Em relação às temperaturas alternadas, observou-se que os resultados obtidos não diferiram ou foram inferiores aos obtidos em $25^{\circ} \mathrm{C}$. Resultado que está de acordo com o trabalho de Alves, Godoy e Correa (2011), onde a germinação de $H$. undatus foi favorecida pela temperatura constante de $25^{\circ} \mathrm{C}$ em relação à temperatura alternada $\left(20-30{ }^{\circ} \mathrm{C}\right)$.

Diversos autores verificaram que a temperatura ótima para a germinação de sementes de diversas espécies de cactos é, normalmente, em torno de $25^{\circ} \mathrm{C}$ (NOBEL, 1988; ROJAS-ARÉCHIGA; VÁZQUEZYANES, 2000; LONE et al., 2007), podendo ocorrer exceções, como para o cacto colunar Oreocereus trolii, que apresenta temperatura ótima à $15{ }^{\circ} \mathrm{C}$ (ZIMMER, 1969), para Schlumbergera truncata (flor-de-maio) na qual a temperatura ótima é 20 ${ }^{\circ} \mathrm{C}$ (LONE et al., 2010) e Pereskia aculeata, um cacto com folhas e porte primitivo, que apresenta germinação ótima à $33{ }^{\circ} \mathrm{C}$ (DAU; LABOURIAU, 1974).

\section{Conclusões}

As temperaturas constantes de 25 e $30{ }^{\circ} \mathrm{C}$ e a alternada de $20-30{ }^{\circ} \mathrm{C}$ são adequadas para a germinação de sementes de $H$. undatus e para o híbrido $H$. undatus x $H$. costaricensis.

Para $H$. polyrhizus, as temperaturas constantes de 20,25 e $30^{\circ} \mathrm{C}$ são adequadas para a germinação das sementes.

A temperatura constante de $25^{\circ} \mathrm{C}$ é a mais adequada para a germinação de sementes de $S$. megalanthus.

Para o híbrido $H$. costaricensis $\mathrm{x} H$. undatus a temperatura constante de $25{ }^{\circ} \mathrm{C}$ e a alternada de $15-25^{\circ} \mathrm{C}$ são adequadas para a germinação das sementes.

\section{Agradecimentos}

À Coordenação de Aperfeiçoamento de Pessoal de Nível Superior (Capes), ao Conselho Nacional de Desenvolvimento Científico e Tecnológico (CNPq) e à Fundação Araucária, pelo apoio financeiro.

\section{Referências}

ALVES, C. Z.; GODOY, A. R.; CORRÊA, L. S. Adequação da metodologia para o teste de germinação de sementes de pitaia vermelha. Ciência Rural, Santa Maria, v. 41, n. 5, p. 779-784, 2011. 
ANDERSON, E. F. The cactus family. Origon: Timber Press, 2001. $776 \mathrm{p}$.

ANDRADE, R. A.; OLIVEIRA, I. V. M.; SILVA, M. T. H.; MARTINS, A. B. G. Germinação de pitaya em diferentes substratos. Revista Caatinga, Mossoró, v. 21, n. 1, p. 71-75, 2008.

BEWLEY, J. D.; BLACK, M. Seeds: physiology of development and germination. 2. ed. New York: Plenum Press, 1994. 445 p.

BRASIL. Ministério da Agricultura e da Reforma Agrária. Regras para análise de sementes. Brasília: SNDA/DNDV/CLAV, 2009. 398 p.

CANTERI, M. G.; ALTHAUS, R. A.; VIRGENS FILHO, J. S.; GIGLIOTI, E. A.; GODOY, C. V. SASM - Agri: sistema para análise e separação de médias em experimentos agrícolas pelos métodos Scott - Knott, Tukey e Duncan. Revista Brasileira de Agrocomputação, Ponta Grossa, v. 1, n. 2, p. 18-24. 2001.

CANTO, A. R. El cultivo de pitahaya en yucatan. Gobierno Del Estado de Yucatan: Universidad Autônoma Chapingo, 1993. 53 p.

CARVALHO, N. M.; NAKAGAWA, J. Sementes: ciência, tecnologia e produção. 4. ed. Jaboticabal: FUNEP, 2000. $588 \mathrm{p}$.

DAU, L.; LABOURIAU, L. G. Temperature control of seed germination in Pereskia aculeata Mill. Anais Academia Brasileira de Ciências, Rio de Janeiro, v. 46, n. 2, p. 311-322, 1974.

GUEDES, R. S.; ALVES, E. U.; GONÇALVES, E. P.; BRUNO, R. L. A.; BRAGA JÚNIOR, J. M.; MEDEIROS, M. S. Germinação de sementes de Cereus jamacaru DC. em diferentes substratos e temperaturas. Acta Scientiarum Biological Sciences, Maringá, v. 31, n. 2, p. 159-164, 2009.

HENDRICKS, S. B.; TAYLORSON, R. B. Variation in germination and amino acid leakage of seed with temperature related to membrane phase change. Plant Physiology, Maryland, v. 58, n. 1, p. 7-11, 1976.

LABOURIAU, L. G. A germinação de sementes. Washington: OEA, 1983. $174 \mathrm{p}$.

LIMA, J. D.; ALMEIDA, C. C.; DANTAS, V. A. V.; SIBA, B. M.; MORAES, W. S. Efeito da temperatura e do substrato na germinação de sementes de Caesalpinia ferrea Mart. ex Tul. (Leguminosae, Caesalpinoideae). Revista Árvore, Viçosa, v. 30, n. 4, p. 513-518, 2006.
LONE, A. B.; SOUZA, G. R. B.; OLIVEIRA, K. S.; TAKAHASHI, L. S. A.; FARIA, R. T. Temperatura e substrato para germinação de sementes de flor-de-maio (Schlumbergera truncata (Haw.) Moran). Revista Ceres, Viçosa, v. 57, n. 3, p. 367-371, 2010.

LONE, A. B.; TAKAHASHI, L. S. A.; FARIA, R. T.; UNEMOTO, L. K. Germinação de Melocactus bahiensis (Cactaceae) em diferentes substratos e temperaturas. Scientia Agraria, Curitiba, v. 8, n. 4, p. 365-369, 2007.

MAGUIRE, J.B. Speed of germination-aid in selection and evaluation for seedling emergence vigor. Crop Science, Madison, v. 2, n. 2, p. 176-177, 1962.

MALUF, A. M.; MARTINS, P. S. Germinação de sementes de Amaranthus hybridus L. e Amaranthus viridis L. Revista Brasileira de Biologia, São Carlos, v. 51, n. 1, p. 417-425, 1991.

MARCOS FILHO, J. Germinação de sementes. In: (Ed.). Semana de atualização em sementes. Campinas: Fundação Cargill, 1986. p. 11-39.

MIZRAHI, Y. A.; NERD, A.; NOBEL, P. S. Cacti as crops. Horticultural Review. New York, v. 18, n. 1, p. 291-320, 1997.

NERD, A.; TEL-ZUR, N.; MIZRAHI, Y. Fruit of vine and columnar cacti. In: NOBEL, P. S. (Ed.). Cacti: biology and uses. Los Angeles: UCLA, 2002. p. 254-262.

NOBEL, P. S. Environmental biology of agaves and cacti. New York: Cambridge University Press, 1988. 270 p.

RILEY, G. J. P. Effects of high temperature on protein synthesis during germination of maize (Zea mays L.). Planta, Berlim, v. 151, n. 1, p. 75-80, 1981.

ROJAS-ARÉCHIGA, M.; VÁZQUEZ-YANES, C. Cactus seed germination: a review. Journal of Arid Environments, London, v. 44, n. 1, p. 85-104, 2000.

SIMÃO, E.; SOCOLOWSKI, F.; TAKAKI, M. The epiphytic cactaceae Hylocereus setaceus (Salm-Dick ex DC.) ralf bauer seed germination is controlled by light and temperature. Brazilian Archives of Biology and Technology, Curitiba, v. 50, n. 4, p. 655-662, 2007.

VAILLANT, F.; PEREZ, A.; DAVILA, I.; DORNIER, M.; REYNES, M. Colorant and antioxidant properties of red pitahaya (Hylocereus sp.). Fruits, Paris, v. 60, n. 1, p. 1-7, 2005.

ZIMMER, K. About the germination of cactus seeds: swelling and germination at constant temperatures. Kakteen und andere Sukkulenten, Stuttgart, v. 20, n. 1, p. 105-107, 1969. 
